\title{
Drift-free attitude estimation for accelerated rigid bodies ${ }^{\text {th }}$
}

\author{
Henrik Rehbinder ${ }^{\mathrm{a}}$, Xiaoming $\mathrm{Hu}^{\mathrm{b}, *}$ \\ ${ }^{a}$ RaySearch Laboratories, Stockholm 111 34, Sweden \\ ${ }^{\mathrm{b}}$ Optimization and Systems Theory, Royal Institute of Technology, Stockholm 10044, Sweden \\ Received 14 January 2003; received in revised form 8 July 2003; accepted 6 November 2003
}

\begin{abstract}
In this paper we study the attitude estimation problem for an accelerated rigid body using gyros and accelerometers. The application in mind is that of a walking robot and particular attention is paid to the large and abrupt changes in accelerations that can be expected in such an environment. We propose a state estimation algorithm that fuses data from rate gyros and accelerometers to give long-term drift free attitude estimates. The algorithm does not use any local parameterization of the rigid body kinematics and can thus be used for a rigid body performing any kind of rotations. The algorithm is a combination of two non-standard, but in a sense linear, Kalman filters between which a trigger based switching takes place. The kinematics representation used makes it possible to construct a linear algorithm that can be shown to give convergent estimates for this nonlinear problem. The state estimator is evaluated in simulations demonstrating how the estimates are long-term stable even in the presence of gyro drift.
\end{abstract}

(C) 2003 Elsevier Ltd. All rights reserved.

Keywords: Attitude estimation; Nonlinear filtering; Multisensor systems; Mobile robots; Sensor fusion

\section{Introduction}

A prerequisite for mobile robot control is state estimation where the states typically are position, velocity and orientation. State estimation is especially important for walking robots in difficult terrain where a sense of balance is absolutely necessary as it is the basis of attitude control. With attitude we refer to the robot's orientation relative to the gravity vector, usually described by pitch and roll. Perhaps most important to walking robots, the problem still applies to any kind of robot moving in difficult terrain. Attitude estimation is usually performed by combining measurements from three kinds of sensors: rate gyros, inclinometers and accelerometers. It is possible to use a rate gyro to derive attitudes by integrating the rigid body kinematic equations. With high-quality gyros and good initial values these estimates can be very accurate over long periods of time. However, if the aim is an autonomous vehicle then the attitude estimate should be reliable over an infinite time scale. It

\footnotetext{
This paper was not presented at any IFAC meeting. This paper was recommended for publication in revised form by Associate Editor Johan Schoukens under the direction of Editor Torsten Söderström.

* Corresponding author.

E-mail addresses: henrik.rehbinder@raysearchlabs.com (H. Rehbinder), hu@kth.se (X. Hu).
}

would also be desirable to use cheap gyros as high-quality gyros are expensive. To provide an absolute reference of the attitude, inclinometers and accelerometers which relate the body to the gravity vector can be used. A problem is that both these sensors also are sensitive to translational accelerations and should only be used during phases of low accelerations. Fundamental for walking robots is that the body motion is inherently three dimensional, making most kinematics representations nonlinear. For nonlinear problems, there are no general state estimation algorithms that are guaranteed to work. Especially Extended Kalman filters (EKF) are known to have an unpredictable behavior even though they often can be used successfully. Attitude estimation via different ensembles of the above mentioned sensors has been studied by many authors such as Vaganay, Aldon, and Fournier (1993), Barshan and Durrant-Whyte (1995), Foxlin (1996), Foxlin, Harrington, and Altshuler (1998), Baerveldt and Klang (1997), Balaram (2000) and by Rehbinder and $\mathrm{Hu}(2000 \mathrm{a}, \mathrm{b})$. Rehbinder and $\mathrm{Hu}$ (2000a, b) have previously designed an algorithm (Rehbinder $\& \mathrm{Hu}, 2000 \mathrm{a}, \mathrm{b}$ ) for fusing inclinometer and gyro data that could be shown to be stable and convergent. However in Rehbinder and $\mathrm{Hu}$ (2000a, b), it was forced to assume low translational accelerations, something that is of course not very realistic for a walking robot. 
In this paper we will provide a solution to fusing data from a 3-axis rate gyro and a 3-axis accelerometer that will provide stable estimates of the robots attitude. The algorithm is based on a switching architecture consisting of two modes, one when accelerations are low, and one when these are high. Given some natural conditions on the motion, the attitude estimates can be shown to converge to the true states. By using a global description of rigid-body rotation we are able to obtain a linear problem and can use a modified linear Kalman filter. As the representation is global (this will be explained in Section 2.1), there are no restrictions on the kind of motions the robot is allowed to perform, contrary to what would be the case if for example Euler angles were used. The contributions of this article are: a mathematically sound, partly linear and quite simple algorithm that solves the important problem of fusing accelerometer and gyro data; The use of a global kinematics representation and a careful treatment of the effect of a switching architecture. The outline of the paper is as follows. In Section 2 the problem is formulated mathematically and the proposed algorithm is described and analyzed. In Section 3 we describe some illustrative simulations first, then present an experiment on a walking robot. The paper is concluded in Section 4 with a discussion and summary.

\section{Problem formulation and solution}

There is one major question to be considered when designing an algorithm for rigid-body rotations and that is what representation to use. As is well known, rigid-body kinematics have a somewhat delicate structure as the rotations is most naturally described as elements of the manifold $S O(3)$. It is common practice to consider some parameterization of $S O(3)$ such as various versions of Euler angels or quaternions. In this study we will work directly with the rotation matrix representation as this, in a sense that will be made clear, will enable a linear formulation and solution. Apart from the above the problem of distinguishing between inertial forces and the gravity vector should be discussed. We will use an accelerometer as the attitude sensor. The problem is of course that when the body is accelerated, the accelerometer is a very bad attitude sensor. We have investigated an approach to the problem that is based on a switching architecture consisting of two modes, one for low accelerations and one for high. It would probably be possible to use a smoothed version of this where the noise parameters are tuned according to the estimated acceleration level. For a study more along this line of thinking we refer to the work by Balaram (2000).

\subsection{Mathematical modeling}

Consider a rigid body moving in inertial space. The body is undergoing both rotations and translations and our aim is to, given a 3 -axis rate gyro and a 3 -axis accelerometer, estimate attitude. Introduce a coordinate system, $N$, fixed in inertial space and a coordinate system, $B$, fixed in the body. Let the coordinates of an arbitrary point be denoted by $\xi^{N}$ if expressed in the $N$-frame and by $\xi^{B}$ if expressed in the $B$-frame. If the vector $r^{N}$ from $0^{N}$ to $0^{B}$ is denoted by $p$ (for position) then the relation between the two frames is $\xi^{B}=R\left(\xi^{N}-p\right)$, where $R$ is a rotation matrix, that is $R^{\prime} R=I$, $\operatorname{det} R=1$. The kinematics of the rigid body (Sastry, 1999) are

$\left\{\begin{array}{l}\ddot{p}=u, \\ \dot{R}=S(\omega) R,\end{array}\right.$

where $u$ is the acceleration expressed in the $N$-frame and where

$S(\omega)=\left(\begin{array}{ccc}0 & \omega_{3} & -\omega_{2} \\ -\omega_{3} & 0 & \omega_{1} \\ \omega_{2} & -\omega_{1} & 0\end{array}\right)$.

$\omega_{i}$ are the components of the angular velocity expressed in the $B$-frame. A rate gyro measures the angular velocity $\omega$ up to an unknown and slowly drifting bias. We will neglect the gyro drift in the analysis but in simulations we will see that the algorithms will work even with gyro biases. An accelerometer measures the sum of inertial forces and gravity. As the accelerometer in this application is assumed to be fixed to the body, these measurements take place in the body frame. If the output from the accelerometer is denoted by $y$ we have that $y=R\left(u-g^{N}\right)$. The idea is to consider the accelerations $u$ as disturbances that we can measure partially through the accelerometer. Take now the $\operatorname{yaw}(\psi)-\operatorname{pitch}(\theta)-\operatorname{roll}(\phi)$ parameterization of $R$, that is

$R=$

$$
\times\left(\begin{array}{ccc}
\mathrm{c} \psi \mathrm{c} \theta & \mathrm{s} \psi \mathrm{c} \theta & -\mathrm{s} \theta \\
-\mathrm{s} \psi \mathrm{c} \theta+\mathrm{c} \psi \mathrm{s} \theta \mathrm{s} \phi & \mathrm{c} \psi \mathrm{c} \phi+\mathrm{s} \psi \mathrm{s} \theta \mathrm{s} \phi & \mathrm{c} \theta \mathrm{s} \phi \\
\mathrm{s} \psi \mathrm{s} \phi+\mathrm{c} \psi \mathrm{s} \theta \mathrm{c} \phi & \mathrm{s} \psi \mathrm{s} \theta \mathrm{c} \phi-\mathrm{c} \psi \mathrm{s} \phi & \mathrm{c} \theta \mathrm{c} \phi
\end{array}\right)
$$

where $\mathrm{c} \psi=\cos \psi$, etc. Note that the third column is independent of yaw and that if that column could be estimated, then it would be possible to extract pitch and roll from it. Denote the $i$ th column of $R$ by $r_{i}(i=1,2,3)$ and let $x=r_{3}$ and note that of course $g^{N}=-g e_{3}$ where $g=9.81 \mathrm{~m} / \mathrm{s}^{2}$, the gravitational constant and where $e_{3}$ is the third unit vector. Now, note that the rotation kinematics also could have been written column wise as $\dot{r}_{i}=S(\omega) r_{i}$. If we for notational simplicity redefine $y:=y / g, u:=u / g$ we have the underlying system

$\left\{\begin{array}{l}\dot{x}=S(\omega) x,\|x(0)\|=1, \\ y=x+R u .\end{array}\right.$

$R u$ is going to be considered as an unknown disturbance. The constraint on the initial condition $\|x(0)\|=1$ is due to 
that $x$ is a column of a rotation matrix and thus has to be of unit length. If $\|x(0)\|=1$ then it will be guaranteed that $\|x(t)\|=1$ as $\left\{x \in \mathbb{R}^{3}:\|x\|=1\right\}$ is a so called invariant manifold for the system $\dot{x}=S(\omega) x$.

Remark. An alternative way to model the pitch and roll dynamics is to use Euler angles. In that way we would end up with a two-dimensional highly nonlinear system. The method we use here is to embed the nonlinear dynamics into a higher dimensional linear one. As a trade-off, the system will evolve on a nonlinear manifold.

What really has to be kept in mind is that when designing an observer for $x$ it must in some way be guaranteed that the state estimates $\hat{x}$ actually do satisfy $\|\hat{x}\|=1$. We will see that the linear structure can be used when designing the attitude estimator as it will turn out that a linear Kalman filter can be used. Clearly, our estimator will be implemented in discrete time. If we denote the underlying sample time by $h$ and assume that $\omega(t)=\omega_{k}$ for $t \in[k h, k h+h)$ then we have the discrete time model

$\left\{\begin{array}{l}x_{k+1}=A_{k} x_{k}, \\ y_{k}=x_{k}+R u_{k},\end{array}\right.$

where $A_{k}=\mathrm{e}^{S\left(\omega_{k}\right) h}$ has a closed form solution given by Rodrique's formula (Sastry, 1999) $A_{k}=I-S\left(\omega_{k}\right) \sin \left(\omega_{k} h\right) /$ $\left\|\omega_{k} h\right\|+S^{2}\left(\omega_{k}\right)\left(1-\cos \left(\omega_{k} h\right)\right) /\left\|\omega_{k} h\right\|^{2}$. Note that the sampled data representation still has $\|x\|=1$ as an invariant manifold. As a matter of fact, $A_{k}=\mathrm{e}^{S\left(\omega_{k}\right) h}$ is a rotation matrix and thus preserves vector lengths.

\subsection{Estimation under low acceleration}

Consider now the idealized case when the accelerations are so low that we can consider them as zero. We might also introduce two noise sources into the model, a process noise $v$ incorporating inaccuracies in modeling and gyro noise and a measurement noise $w$ which models accelerometers noise as well as high-frequency accelerations. The system that we use as a model in the filtering equations is

$\left\{\begin{array}{l}x_{k+1}=A_{k} x_{k}+v_{k}, \\ y_{k}=x_{k}+w_{k},\end{array}\right.$

where the covariance matrices for $v_{k}$ and $w_{k}$ are $Q$ and $R$. Note one very important feature, $\|x\|=1$ is no longer an invariant manifold to (6). This might appear undesirable but model (6) is just used for designing the observer. The noise covariance matrices $Q$ and $R$ are to be regarded as tuning parameters. Model (4) is what we consider as the "true" underlying system. The constraint that $\left\|x_{k}\right\|=1$ will be incorporated later. At present, we have a linear time-varying system model and for such, a linear standard Kalman filter can be used. Such a Kalman filter will be well behaved in itself due to the linearity of the underlying system. The estimates produced will not have unit length but this is taken care of in an extra step outside the filtering equations. To summarize, a linear Kalman filter computes state estimates $\hat{z}_{k}$ that are not of unit length and these estimates are used to obtain the state estimate $\hat{x}_{k}=\hat{z}_{k} /\left\|\hat{z}_{k}\right\|$ which has unit length. For simplicity, we take the state predictor as the formulas are more streamlined. The estimation algorithm under low accelerations is thus

$\hat{z}_{k+1}=A_{k} \hat{z}_{k}+K_{k}\left(y_{k}-\hat{z}_{k}\right)$,

$K_{k}=A_{k} P_{k}\left(P_{k}+R\right)^{-1}$,

$P_{k+1}=A_{k} P_{k} A_{k}^{\prime}+Q-A_{k} P_{k}\left(P_{k}+R\right)^{-1} A_{k}^{\prime} P_{k}$,

$\hat{x}_{k}= \begin{cases}\hat{z}_{k} /\left\|\hat{z}_{k}\right\| & \text { if } \hat{z}_{k} \neq 0, \\ \hat{z}_{k-1} & \text { if } \hat{z}_{k}=0 .\end{cases}$

\subsection{Estimation under high acceleration}

When the acceleration is so high that the accelerometer must be considered completely unreliable as a gravity sensor we are forced to rely on the rate gyros. Integrating these can be obtained by simply setting the accelerometer noise covariance matrix $R$ to infinity. We then get the algorithm for pitch and roll estimation under high accelerations

$\hat{z}_{k+1}=A_{k} \hat{z}_{k}$,

$P_{k+1}=A_{k} P_{k} A_{k}^{\prime}+Q$,

$\hat{x}_{k}= \begin{cases}\hat{z}_{k} /\left\|\hat{z}_{k}\right\| & \text { if } \hat{z}_{k} \neq 0, \\ \hat{z}_{k-1} & \text { if } \hat{z}_{k}=0 .\end{cases}$

\subsection{Acceleration detection}

In order to handle the problem of attitude estimation under accelerations it would be valuable to be able to detect acceleration or rather, to detect non-accelerations. Consider now the case when $u=0$ and study what necessary conditions on the accelerometer signal that holds for an acceleration free movement. Recall that the accelerometer model is $y=x+R u$, (where now $u=0$ ) so for the accelerometer signal magnitude it must hold that $s(t)=\|y(t)\|^{2}-1=\|x\|^{2}-1=0$. This condition is nothing but a formulation of the fact that when $u=0$, then the accelerometer output (viewed as a vector) is moving on a sphere of radius 1 . We state the above as an observation.

Observation 2.1. A necessary condition for acceleration free movements is

$\|y(t)\|^{2}=1$.

The idea is now to use the necessary conditions to design the switching rule when switching between the two modes 


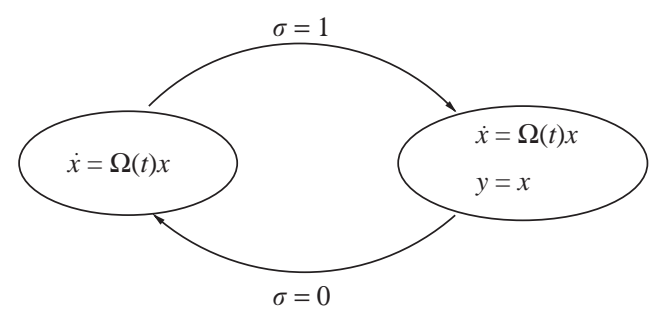

Fig. 1. Switching architecture.

described above. It is then crucial to understand what might go wrong, that is, what kind of non-zero accelerations will result in the output $\|y(t)\|^{2}=1$. We have

$$
\begin{aligned}
1 & =\|y\|^{2}=x^{\prime} x+u^{\prime} R^{\prime} R u+2 x^{\prime} R u \\
& =1+\|u\|^{2}+2 e_{3}^{\prime} u=1+\|u\|^{2}+2 u_{3},
\end{aligned}
$$

where we have used that $x=R e_{3}$ and $R^{\prime} R=I$. So, the accelerations that might cause false triggers are those that satisfy $u_{1}^{2}+u_{2}^{2}+\left(u_{3}+1\right)^{2}=1$ and therefore live on a sphere. It is quite clear that acceleration trajectories that move on this sphere are very rare. However, not only those acceleration trajectories that evolve on the sphere will produce false triggers, also those that pass through it will. We must therefore assure that the switching rule is such that we only switch when it is highly unlikely that the accelerations are anything but zero. Such a rule is to demand $\|y\|=1$ for a certain amount of time $T_{0}$ and this is the approach that we will use. It should be pointed out that this idea has been proposed earlier by Foxlin (1996) and Foxlin et al. (1998).

\subsection{Hybrid estimation — switching}

The detection rule we will use is coded by the binary variable $\sigma$ defined by

$\sigma(t)= \begin{cases}1, & \|y(\tau)\|=1 \forall \tau \in\left[t-T_{0}, t\right], \\ 0 & \text { otherwise }\end{cases}$

and is to be interpreted as: $\sigma=1$ if accelerations are estimated low and 0 otherwise. $\sigma$ will be denoted the switching path (Dayawansa \& Martin, 1999). Using this function, the combined filter can be conveniently written as

$\hat{z}_{k+1}=A_{k} \hat{z}_{k}+\sigma\left(t_{k}\right) K_{k}\left(y_{k}-\hat{z}_{k}\right)$,

$K_{k}=\sigma\left(t_{k}\right) A_{k} P_{k}\left(P_{k}+R\right)^{-1}$,

$P_{k+1}=A_{k} P_{k} A_{k}^{\prime}+Q-\sigma\left(t_{k}\right) A_{k} P_{k}\left(P_{k}+R\right)^{-1} P_{k} A_{k}^{\prime}$,

$\hat{x}_{k}= \begin{cases}\hat{z}_{k} /\left\|\hat{z}_{k}\right\| & \text { if } \hat{z}_{k} \neq 0, \\ \hat{z}_{k-1} & \text { if } \hat{z}_{k}=0,\end{cases}$

where the switching between the two modes indicated in Fig. 1 is encoded with the $\sigma$ function. The algorithm proposed can be shown to be convergent given some rather mild and reasonable conditions on the accelerations. That convergence is not trivially guaranteed can be understood when considering the underlying systems that were used to design the two Kalman filters. System (6) is observable (the Kalman filter would converge if this was the only mode used) and system (8) is unobservable (the filter would diverge). Now we are switching between these two different filters and it is not obvious that the combined will converge. However, the following holds "If the system model upon which the Kalman filter is based is stochastically observable and stochastically controllable, then the filter is uniformly asymptotically globally stable" (Maybeck, 1979), that is, the estimation errors go to zero. The switching system model can be written as

$\left\{\begin{array}{l}x_{k+1}=A_{k} x_{k}+v_{k}, \\ y_{k}=C_{k} x_{k}+w_{k},\end{array}\right.$

where $C_{k}=\sigma\left(t_{k}\right) I$. The covariance matrix of $v_{k}$ is a tuning parameter at our disposal and it can be taken as a diagonal matrix with non-zero entries. Then each state can be independently controlled and the system can easily be shown to be stochastically controllable. As to observability, we will have to impose some restrictions on the switching path/accelerations. Let the switching times for the switching path be denoted by $t_{n}^{0}$ and $t_{n}^{1}$ according to

$\sigma(t)= \begin{cases}0, & t_{n}^{0} \leqslant t<t_{n}^{1} \text { for some } n, \\ 1, & t_{n}^{1} \leqslant t<t_{n+1}^{0} \text { for some } n,\end{cases}$

Definition 2.1. $\sigma(t)$ is called non-pathological if $\exists T_{l}>0$ such that

$\inf _{n}\left(t_{n+1}^{0}-t_{n}^{1}, t_{n}^{1}-t_{n}^{0}\right) \geqslant T_{l}$.

$\sigma(t)$ is called non-degenerate if $\exists T_{u}<\infty$ such that

$\sup _{n}\left(t_{n}^{1}-t_{n}^{0}\right) \leqslant T_{u}$.

Non-pathological switching means simply that switching is such that there is a lower bound on the minimum time that the system is in each mode (so chattering is avoided) and non-degeneracy that there is an upper bound on the time that the systems remains in the unobservable mode.

Observation 2.2. If $\sigma$ is non-pathological and nondegenerate then (12) is stochastically observable.

Proof. Stochastic observability amounts to checking if there exists $\alpha, \beta$ : $0<\alpha<\beta<\infty$ and a positive integer $N$ such that for all $i \geqslant N$

$\alpha I \leqslant \sum_{j=i-N+1}^{i} \Phi_{j, i}^{\prime} C_{j}^{\prime} R^{-1} C_{j} \Phi_{j, i} \leqslant \beta I$,

where $\Phi_{j, i}=A_{j-1} \ldots A_{i}$. Here $C_{j}=I \sigma\left(t_{j}\right)$. Take an arbitrary $z \in \mathbb{R}^{3}$ and consider $\phi(z)=\sum_{j=i-N+1}^{i} z^{\prime} \Phi_{j, i}^{\prime} C_{j}^{\prime} R^{-1} C_{j} \Phi_{j, i} z$. If now $N>T_{u} / T_{l}$ then there will be at least one element of the 
sum (say the $j_{k}$ th element) where $\sigma\left(t_{j_{k}}\right)=1$. That such an $N$ exist follows from that the switching is non-pathological and non-degenerate. Now

$\phi(z) \geqslant z^{\prime} \Phi_{j_{k}, i}^{\prime} R^{-1} \Phi_{j_{k}, i} z \geqslant \operatorname{mineig}\left(R^{-1}\right)\|z\|^{2} \geqslant \alpha\|z\|^{2}$,

where the second inequality is due to the fact that $\Phi$ is a rotation matrix which is an isometric mapping and elementary linear algebra. As $R$ is positive definite, the existence of $\alpha>0$ is proven. That $\phi(z)$ is bounded from above for bounded $z$ is apparent from the isometric properties of $\Phi$.

\subsection{Filter analysis}

It turns out that the filter equations can be simplified for a clever choice of the tuning parameters $Q$ and $R$, namely that $Q=q I$ and $R=r I$. Due to the fact that $A$ is an orthogonal matrix it can be shown that the Riccati equation can be described by a scalar equation.

Observation 2.3. If $Q=q I, R=r I, P_{0}=p_{0} I$ and $A_{k} A_{k}^{\prime}=I$, then $P_{k}=p_{k} I$ where $p_{k+1}=p_{k}+q-\sigma_{k} p_{k}^{2} /\left(p_{k}+r\right)$.

The proof is just a simple calculation. We know that $P_{0}=$ $p_{0} I$. Now assume that $P_{k}=p_{k} I$. Then

$$
\begin{aligned}
P_{k+1} & =A_{k} p_{k} I A_{k}^{\prime}+q I-\sigma_{k} A_{k} p_{k} I\left(p_{k} I+r I\right)^{-1} p_{k} I A_{k}^{\prime} \\
& =A_{k} A_{k}^{\prime}\left(p_{k}-\sigma_{k} \frac{p_{k}^{2}}{p_{k}+r}\right)+q I \\
& =\left(p_{k}+q-\sigma_{k} \frac{p_{k}^{2}}{p_{k}+r}\right) I .
\end{aligned}
$$

The proof follows from induction.

Now, the filter equations can be written in the more condensed form

$\hat{z}_{k+1}=A_{k}\left[\hat{z}_{k}+\sigma\left(t_{k}\right) \frac{p_{k}}{p_{k}+r}\left(y_{k}-\hat{z}_{k}\right)\right]$,

$p_{k+1}=p_{k}+q-\sigma_{k} \frac{p_{k}^{2}}{p_{k}+r}$,

$\hat{x}_{k}= \begin{cases}\hat{z}_{k} /\left\|\hat{z}_{k}\right\| & \text { if } \hat{z}_{k} \neq 0, \\ \hat{z}_{k-1} & \text { if } \hat{z}_{k}=0\end{cases}$

and it is clear that this is quite simple to implement. The only matrix operations involved are those involving $A_{k}$. The Riccati equation is also scalar.

\section{Simulations and experiments}

We will demonstrate the filter algorithms using numerical simulations. The simulations are meant to resemble a moving robot that once in a while stops (enters a non-accelerated
Table 1

Parameter values used in the simulations

\begin{tabular}{llll}
\hline Gyro noise & Accm noise & Gyro offset & $T_{\mathrm{s}}$ \\
\hline $0.01(\mathrm{std})$ & $0.1(\mathrm{std})$ & 0.1 & 0.01 \\
\hline
\end{tabular}

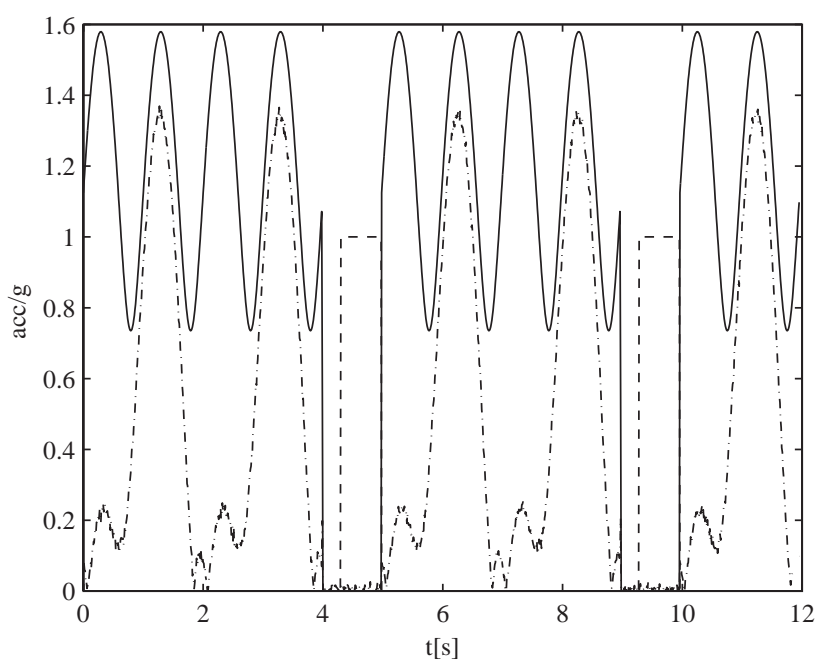

Fig. 2. Switch signals. Solid-acceleration magnitude, dash-dottedaccelerometer magnitude, dashed-switch signal.

mode). The motion considered is such that the switching structure is both non-pathological and non-degenerate. Continuous time motion and sensor data were generated and the sensor data were sampled with a sampling frequency of $100 \mathrm{~Hz}$ after which white noise was added. Also, a rate gyro offset was added to ensure realistic simulations. To demonstrate that the proposed filter actually is convergent it was initialized with an erroneous guess. The above parameter values are summarized in Table 1 . The simulations were performed using SIMULINK on an Ultra Sparc 5 workstation. With imperfect and noisy sensors (as in the simulations) the switching rule $\|y\|-1=0$ can obviously not be used. It is natural to use thresholding instead and the rule

$\sigma(t)=\left\{|\|y(\tau)\|-1| \leqslant \varepsilon, \quad \forall \tau \in\left[t, t-T_{0}\right]\right\}$

was used instead. $\varepsilon$ was chosen as three standard deviations of the accelerometer noise. The time threshold is hard to give a general rule for how it should be chosen. We argue that it would be very unlikely for the robot to maintain a false alarm acceleration for $T_{0}=0.2 \mathrm{~s}$ and this was the method used in the simulations. The only reasonable way of choosing $T_{0}$ is through extensive testing for the application in mind.

The results of a $12 \mathrm{~s}$ simulations is shown in Figs. 2-4. In order to simplify the tuning, the matrices $Q, R, P_{0}$ are chosen as discussed in Section 2.6, with only parameters $q, r, p_{0}$ to tune. The simulations have been run for $100 \mathrm{~s}$ and the $12 \mathrm{~s}$ simulations do describe the interesting features. The motion considered consists of $4 \mathrm{~s}$ of high accelerations and $1 \mathrm{~s}$ of zero acceleration repeated periodically. In Fig. 2 three graphs 

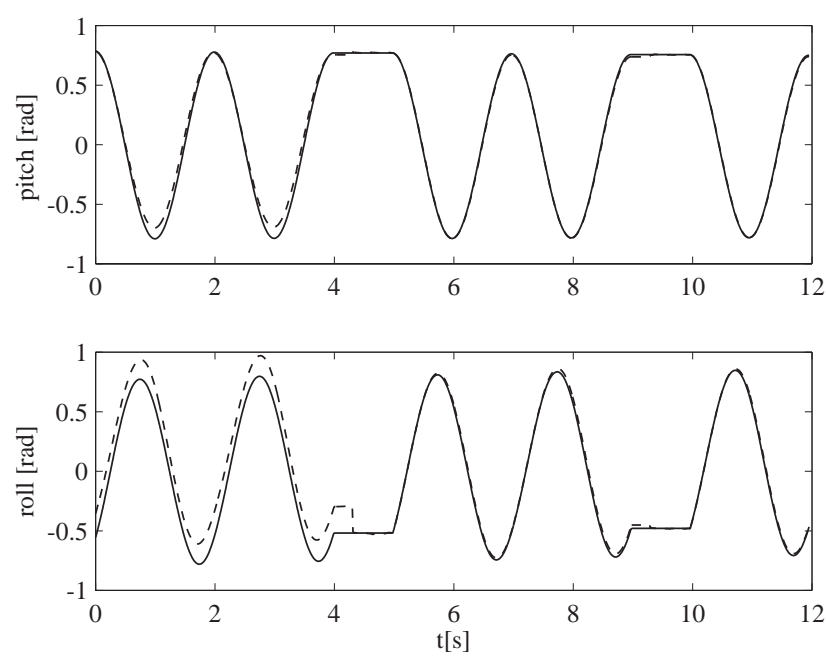

Fig. 3. Pitch (top) and roll (bottom) angles. Solid lines denote true values and dashed estimated.
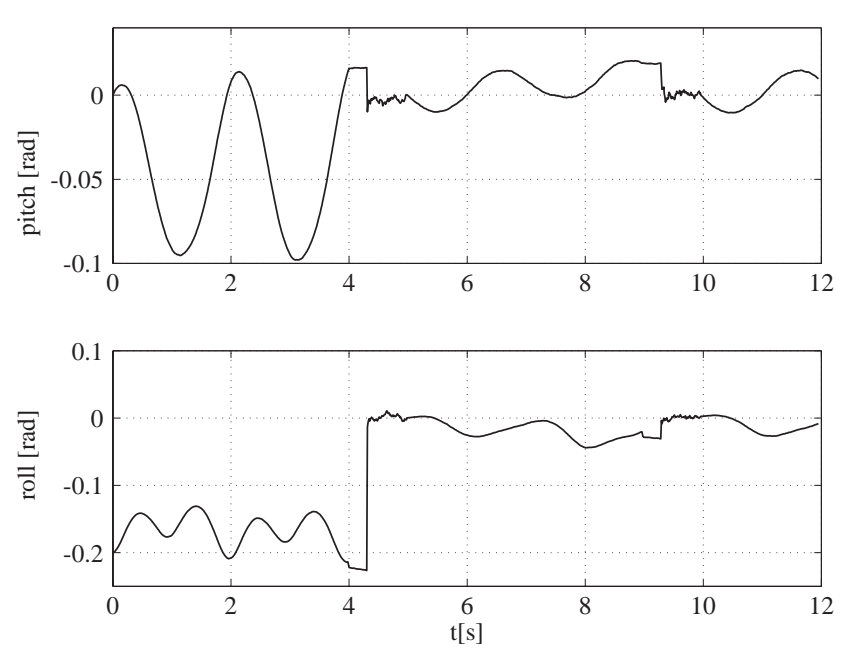

Fig. 4. Pitch and roll errors.

concerning the switching signals are shown. The solid line is the magnitude of the true acceleration and it is clear how it abruptly changes between the high and low mode. The dashdotted line is $s(t)=|\|y(t)\|-1|$, that is, the signal which is used to detect low accelerations. It is apparent from the graph that it is crucial to use for example the time window approach as there are several occasions, where $s(t)=0$ even though the true acceleration is non-zero. Finally, the dashed line is the switch signal $\sigma(t)$. Using this switch signal, the described Kalman filter is run to estimate $x(t)$. In Fig. 3 the estimates, expressed in pitch/roll are shown. As the filter was initialized with an erroneous initial guess, there is a large error for the first $4 \mathrm{~s}$ when the body is accelerated. As soon as the filter goes into the low-acceleration mode the errors rapidly decrease. A complementary view of the errors is given in Fig. 4 where the effect of the unmodeled gyro offsets is clear. A small trend of increasing errors during

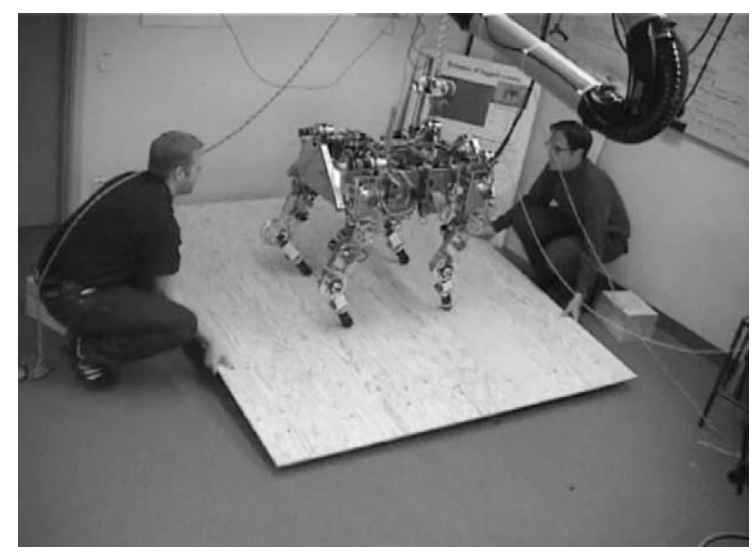

Fig. 5. Balance control of a walking robot: www.math.kth.se/ hu/ balance.mpg.

the high-acceleration phases is apparent but these errors are compensated for during the low-acceleration phase. It should be pointed out that the gyro offsets chosen in this study are rather high and would correspond to very badly calibrated gyros.

Finally, we should point out that the filter has been successfully implemented on a walking robot (Ridderström, 2003 ) in closed loop, namely the estimation is used in the feedback control of body balance (Fig. 5). In this experiment, the walking robot was placed on a balancing board which was made to jerk back and forth with quite rapid movements. The attitude estimator described in this paper provided the estimates that the balance controller used for keeping the robot trunk parallel to the floor.

\section{Summary and discussion}

In this paper we have presented an approach to solving the attitude estimation problem for a rigid body. The algorithm does in a theoretically consistent and easily implementable way solve this problem. The application in mind is a walking robot but the algorithms are in no way dependent on that application and could just as well be applied to other robots such as flying or climbing ones. For climbing robots, the use of the global representation instead of the more standard yaw/pitch/roll parameterization could prove very useful as such robots usually have an attitude out of the bounds for which that parameterization is valid. The kinematics representation is very important as the linear Kalman filter can be used which makes it possible to easily obtain theoretical convergence results. It is worth emphasizing that the hard nonlinear problems concerning these problems stem from the choice of coordinates and that it was possible to avoid them by a suitable representation. The approach presented in this paper does not address the problem of estimating the entire rotation matrix $R$. To be able to do this, some additional sensor has to be used. The use of vision together with rate gyros has been studied by Rehbinder and Ghosh (2003). 
One error source in the filter is due to the gyro bias. It would in theory be possible to design a gyro bias estimator. Such bias estimates would however be very sensitive to disturbances from accelerations. In fact, we have experimented with this idea, but we could not achieve an acceptable performance. In fact, due to the sensitivity the performance with bias estimation was often worse than without.

\section{Acknowledgements}

This work was in part sponsored by the SSF through its Centre for Autonomous Systems at KTH and in part by VR.

\section{References}

Baerveldt, A. J., \& Klang, R. (1997). A low-cost and low-weight attitude estimation system for an autonomous helicopter. In IEEE international conference on intelligent engineering systems, proceedings, Budapest, Hungary (pp. 391-395).

Balaram, J. (2000). Kinematic observers for articulated rovers. In Proceedings of the 2000 IEEE international conference on robotics and automation, San Francisco (pp. 2597-2604).

Barshan, B., \& Durrant-Whyte, H. F. (1995). Inertial navigation systems for mobile robots. IEEE Transactions on Robotics and Automation, 11(3), 328-342.

Dayawansa, W. P., \& Martin, C. F. (1999). A converse Lyapunov theorem for a class of dynamical systems which undergo switching. IEEE Transactions on Automatic Control, 44(4), 751-760.

Foxlin, E. (1996). Inertial head-tracker sensor fusion by a complementary separate-bias Kalman filter. In Proceedings of the IEEE 1996 virtual reality annual international symposium, Santa Clara, CA (pp. 185-194).

Foxlin, E., Harrington, M., \& Altshuler, Y. (1998). Miniature 6-DOF inertial system for tracking HMD. In Proceedings of the SPIE, Vol. 3362, Orlando, FL (pp. 214-228).

Maybeck, P. S. (1979). Stochastic models, estimation and control, Vol. 1. New York: Academic Press.

Rehbinder, H., \& Ghosh, B. K. (2003). Pose estimation using line-based dynamic vision and inertial sensors. IEEE Transactions on Automatic Control, 48(2), 186-199.
Rehbinder, H., \& Hu, X. (2000a). Nonlinear pitch and roll estimation for walking robots. In Proceedings of the 2000 IEEE international conference on robotics and automation, Vol. 3, San Francisco, CA, USA (pp. 2617-2622).

Rehbinder, H., \& Hu, X. (2000b). Nonlinear state estimation for rigid body motion with low-pass sensors. Systems and Control Letters, 40(3), 183-190.

Ridderström, C. (2003). Legged locomotion: Balance, control and tools. Doctoral thesis, KTH, 2003.

Sastry, S. (1999). Nonlinear systems: Analysis, stability, and control. New York: Springer.

Vaganay, J., Aldon, M., \& Fournier, A. (1993). Mobile robot attitude estimation by fusion of inertial data. In Proceedings of the 1993 IEEE international conference on robotics and automation, Vol. 1 (pp. 277-282).

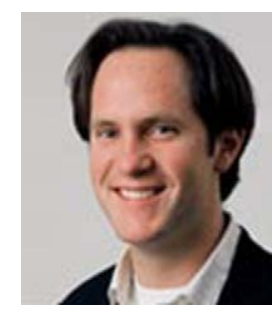

Henrik Rehbinder was born in 1972 in Värmdö, Stockholm, Sweden. He received his M.Sc. degree in Engineering Physics at the Royal Institute of Technology in 1996. He received his Ph.D. degree in 2001 from the Division of Optimization and Systems Theory where he was affiliated with the Centre for Autonomous Systems. His research interests are various applications of control theory to problems in sensor fusion for mobile robots and real-time systems. He is currently director of research at RaySearch Laboratories AB, Stockholm, Sweden and his main responsibility is optimization and control design for radiation therapy for cancer.

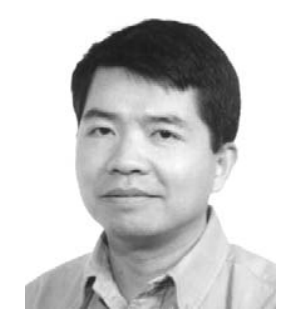

Xiaoming Hu was born in Chengdu, China, in 1961. He received the B.S. degree from University of Science and Technology of China in 1983. He received the M.S. and Ph.D. degrees from Arizona State University in 1986 and 1989 respectively. From 1989 to 1990 he was a Gustafsson Postdoctoral Fellow at the Royal Institute of Technology, Stockholm, where he is currently a professor. His main research interests are in nonlinear feedback stabilization, nonlinear observer design, sensing and active perception, motion planning and control of mobile robots, and mobile manipulation. 